Cite this: Soft Matter, 2014, 10, 357

\title{
Microstructural, mechanical and mass transport properties of isotropic and capillary alginate gels
}

\begin{abstract}
Erich Schuster, ${ }^{\text {ab }}$ Johanna Eckardt, ${ }^{\text {bc }}$ Anne-Marie Hermansson, ${ }^{\text {abd }}$ Anette Larsson, ${ }^{\text {bc }}$ Niklas Lorén, ${ }^{\mathrm{ab}}$ Annika Altskär ${ }^{\mathrm{ab}}$ and Anna Ström ${ }^{* \mathrm{bc}}$

Macroscopically homogeneous and inhomogeneous calcium alginate gels are formed via internal or external addition of various amounts of calcium to an alginate solution. The externally formed gels contain parallel aligned capillary structures. The mechanical and mass transport properties and the microstructure of the differently set gels were characterized by rheological measurements, fluorescence recovery after photobleaching (FRAP) and transmission electron microscopy (TEM). TEM images show a zone of distorted anisotropic gel structure in the vicinity of the capillaries as well as indications of a lower degree of void connectivity. The diffusion rates of dextran at large distances from the capillaries were fast and capillary gels showed a plastic behaviour in comparison to the internally set gels. The results presented show large functional differences between the internally and externally set gels, which cannot be explained by the presence of capillaries alone.

Received 29th August 2013

DOI: $10.1039 / \mathrm{c} 3 \mathrm{sm} 52285 \mathrm{~g}$

www.rsc.org/softmatter
\end{abstract}

The egg box model involves a two-step network formation

\section{Introduction}

Alginate is used as a thickener and gelling agent in diverse fields such as food, ${ }^{1}$ pharmaceutics ${ }^{2-4}$ and waste water treatment. ${ }^{5}$ Besides its use as a thickener and stabilizer in food products, alginate gel is also particularly suitable for biomedical applications $^{2,6}$ - such as tissue engineering ${ }^{7,8}$ and as a material for cell immobilisation and cell signalling.9-11 - due to its high biocompatibility, low cost and a mild gelation process. Alginate has a strong affinity for di- and trivalent cations, and forms rapidly a gel in the presence of low concentrations of such ions $\left(\mathrm{Mg}^{2+}\right.$ being an exception) at a range of $\mathrm{pH}$ values and temperatures. Alginate is mostly derived from brown algae but is also produced by bacteria. It is a charged and linear copolymer consisting of (1-4) linked $\beta$-D-mannuronic acid (M) and $\alpha$-Lguluronic acid $(\mathrm{G})$, whose ratio varies with the alginate source. The physical properties of the alginate depend on the $\mathrm{M} / \mathrm{G}$ ratio as well as the distribution of the $\mathrm{M}$ and $\mathrm{G}$ units along the alginate chain. The ability of alginate to form networks in the presence of di-valent cations, where calcium has been specifically studied, is attributed to chelation of calcium between $\mathrm{G}$ units from different alginate chains via the so-called egg-box model. ${ }^{12}$

${ }^{a}$ Department of Structure and Material Design, The Swedish Institute for Food and Biotechnology, SIK, Göteborg, Sweden

${ }^{b}$ SUMo BIOMATERIALS, VINN Excellence Center, Chalmers University of Technology, Göteborg, Sweden. E-mail: anna.strom@chalmers.se

'Pharmaceutical Technology, Department of Chemical and Biological Engineering, Chalmers University of Technology, Göteborg, Sweden

${ }^{d}$ Department of Applied Surface Chemistry, Chalmers University of Technology, Göteborg, Sweden mechanism where the first step is a dimerization process ${ }^{\mathbf{1 2}}$ followed by dimer-dimer aggregation of $\mathrm{G}$ units and $\mathrm{Ca}^{2+}$, where the degree of aggregation is partly determined by total calcium concentration. ${ }^{13}$ It follows that the total amount of $\mathrm{G}$ units, and its distribution along the chain, is an important aspect for the ion induced gelation ability of alginate, where the high amount of $\mathrm{G}$ units and GG blocks are favourable. ${ }^{2}$ Further, it is believed that G-blocks of 6-10 residues are necessary in order to form stable crosslinked junctions with divalent counterions. Large deformation studies of calcium alginate gels have shown that fracture occurs when enough stress is applied to disrupt the junction zones. Generally, an increasing amount of calcium at a fixed alginate concentration leads to an increased modulus $^{\mathbf{1 4 , 1 5}}$ while the fracture strain is independent of both alginate and calcium concentration. ${ }^{15}$ Dependent on gel composition, large deformation of calcium alginate gels has shown strain hardening properties. ${ }^{\mathbf{1 6}}$

Determination of the thickness of the network strands and the network structure has been done using small angle X-ray scattering (SAXS) ${ }^{\mathbf{1 3}}$ and transmission electron microscopy (TEM). ${ }^{17}$ In general, increasing the amount of calcium leads to an increased modulus and strand thickness. However, SAXS and rheological studies of calcium alginate networks showed further that gels with a similar storage modulus for a given polymer concentration can be obtained using different alginates and calcium concentration combinations and that the strand thickness, or the local structure of alginate gels, varies considerably despite the similar gel modulus. ${ }^{\mathbf{1 3}}$ Determination of strand thickness and the overall network structure of a given alginate concentration at increasing calcium concentrations did 
not reveal apparent network differences using TEM. ${ }^{17}$ Similar observations have been made for calcium pectin, where the network structures visualised using TEM were similarly independent of the calcium concentration despite a clear influence of calcium on the rheological properties of the final gel. ${ }^{18}$ This implies that the concentration of calcium influences molecular interactions within the network strands rather than the overall morphology of the network. ${ }^{19}$

The kinetics of calcium alginate chelation is fast, thus a slow introduction of calcium ions is typically required in order to reduce the risk of precipitation of calcium alginate particles upon addition of calcium or large inhomogeneities within the alginate gel. One common way to introduce calcium ions into the alginate solution is to disperse an insoluble calcium source in the alginate solution, followed by the addition of a slowly hydrolysed acid. ${ }^{13}$ As the acid gets deprotonated over time the calcium salt is solubilised forming a relatively homogeneous network. ${ }^{20}$ Such calcium alginate gels are known to be of isotropic character and the gelation methodology is typically referred to as the internal method., ${ }^{\mathbf{1 2 1}}$ Alginate gelation has also been studied where the alginate solution is e.g. inserted in a dialysis membrane, allowing for diffusion of small molecules into the membrane but not of polymers out of the membrane. Ion-induced gelation performed in such a way revealed an increased concentration of both polymer and crosslinking ions towards the gel surface and a less concentrated polymer and ion concentration towards the center of the gel. ${ }^{22}$

A third gelation methodology involves the anchoring of alginate to the container surface and the creation of a top gelled alginate layer, over which an ion solution composed of ions enabling crosslinks is added. The ions in this way diffuse into the alginate solution, across the gelled membrane, creating highly inhomogeneous and anisotropic alginate gels. Gels formed via this external mechanism were found to form capillaries, from the top of the gel to the bottom. Thiele and co-workers, ${ }^{23}$ who first reported the tendency of alginate to form anisotropic and highly inhomogeneous structures with open capillaries, were intrigued by its resemblance to structures of the biological origin. ${ }^{7}$ The interest for these structures has been enduring due to its potential application in the area of tissue engineering..$^{7,24,25}$

However, the mechanism of capillary formation is still not fully understood. The most developed approach in modelling the behaviour is by Kohler and co-workers, ${ }^{26,27}$ where a Rayleigh-Benard like instability is used to explain the existence and propagation of the capillaries as gel formation progresses. A recent magnetic resonance (MR) microscopy study provided further insights on the dynamics of gel formation and front propagation in the alginate capillary systems. ${ }^{28} \mathrm{MR}$ allowed us to confirm that the gel front has a definite structure ${ }^{22}$ and that the reaction front position $z_{\mathrm{F}}(t)$ can be described by a diffusion driven term plus a pseudo-advective velocity component: $z_{\mathrm{F}}(t)$ $\approx(D t)^{1 / 2}+v t .^{29,30}$ The 'pseudoadvective' component arises from stress relaxation due to polymer swelling in models of solvent diffusion into polymer. Further, Maneval et $a .^{28}$ show that microscale flow processes near a gel front, where capillaries form, are complex and vary with time, and do not seem to support the details of a proposed front propagation and capillary formation model based on dissipative flow structures and
Rayleigh-Benard instabilities. Rather, an alternative theoretical approach based on spinodal decomposition in the presence of anisotropic stress distribution in gels is suggested as a possible mechanism governing the capillary formation. ${ }^{31,32}$

While several studies have been conducted on the theory of the capillary formation and the effect of various crosslinking ions on the capillary formation (diameter and density), as well as the viability of live cells within the capillary alginate gels, no studies have actually investigated the microstructure of such gels and its potential impact on the functionality of the gel: e.g. diffusivity of certain compounds and mechanical strength - compared to the functionality of internally set (isotropic) calcium alginate gels.

For internally set gels both diffusion and mechanical properties have been studied to some extent. The diffusivity of proteins in isotropic alginate gels depends on the guluronic acid content, which is explained in terms of the difference in flexibility of the polymer backbone. ${ }^{33}$ Further techniques utilized in order to study solute diffusion in isotropic alginate gels are NMR diffusometry, ${ }^{17}$ and fluorescence correlation spectroscopy. ${ }^{34}$ Another complementary method for local diffusion measurements is fluorescence recovery after photobleaching (FRAP). In FRAP, the fluorescent molecules are first irreversibly bleached in a limited volume with a short high intensity pulse of light, which results in a local decrease of the fluorescence intensity. After bleaching, unbleached fluorescent molecules from the surroundings gradually diffuse into the bleached region while bleached fluorescent molecules diffuse out. The fluorescence intensity in the bleached region will recover at a rate that depends on the mobility of the molecules. With an appropriate mathematical model one can then extract quantitative information on the molecular dynamics by analyzing the fluorescence recovery. FRAP has established itself in the last decade as a powerful method of measuring the diffusion of fluorescently tagged microspheres, dendrimers, proteins, polysaccharides in soft matter systems, ${ }^{35-37}$ and is reviewed elsewhere. ${ }^{38}$

The aim of the study was to compare the functionality of alginate gels of same composition, but formed via the internal gelation method (leading to isotropic structure) or external addition of calcium. Gels formed via the external methodology form macroscopic pores or capillaries, hence these gels are addressed in due course as capillary gels. The mechanical strength of the gels was compared via stress relaxation and large deformation rupture measurements. The mass transport properties were evaluated via probe diffusion measurements on different molecular weight FITC-dextrans. Further, transmission electron microscopy (TEM) and image analysis were utilized to characterize the microstructure at the nanometre level. The measured mechanical and mass transport properties were related to the microstructure of the gels.

\section{Experimental}

\section{Materials}

The alginate, Protanal RF6650, was kindly provided by FMC Biopolymers, UK. The alginate contains $70 \%$ guluronate according to the supplier. $\mathrm{CaCO}_{3}$ with an average particle size of $10 \mu \mathrm{m}{ }^{39}$ was obtained from Provencale SA, France. 
Glucono- $\delta$-lactone (GDL) was obtained from Sigma - Aldrich, as was $\mathrm{NaCl}, \mathrm{CaCl}_{2}, \mathrm{HCl}$ and $\mathrm{NaOH}$. The fluorescent probes used were FITC-dextran with molecular weights (MWs) of $10 \mathrm{kDa}, 70$ $\mathrm{kDa}$ and $500 \mathrm{kDa}$ (Invitrogen Molecular Probes, U.S.A.). Ruthenium Red was obtained from Sigma-Aldrich Nordic. The secure-seal spacers used were $120 \mu \mathrm{m}$ thick and $9 \mathrm{~mm}$ in diameter (Invitrogen, U.S.A.).

\section{Methods}

Alginate solutions $(2 \% \mathrm{w} / \mathrm{w})$ were prepared by careful addition of alginate powder to deionised water at room temperature under vigorous stirring. The dispersion was thereafter heated to $353 \mathrm{~K}$ in a water bath and kept at this temperature for 30 minutes or until dissolution was obtained. The $\mathrm{pH}$ of the polymeric solution was adjusted from $\mathrm{pH} 7.3$ to $\mathrm{pH} 7$ using $0.1 \mathrm{M} \mathrm{HCl}$.

Gel preparation. Alginate gels using internal setting were prepared by controlled release of calcium. $\mathrm{CaCO}_{3}$ and GDL were rapidly dispersed in water and immediately added to the alginate solution to yield a final alginate concentration of $1.8 \%$ or $2 \% \mathrm{w} / \mathrm{w}$, respectively. The dispersions were poured into cylindrical Teflon moulds ( $h=12.5 \mathrm{~mm} ; d=12.5 \mathrm{~mm})$. The moulds were sealed and the samples were allowed to equilibrate and set at room temperature for $48 \mathrm{~h}$ prior to use. It is important to note that the GDL was always used in stoichiometric equivalence to $\mathrm{CaCO}_{3}$ (e.g. $15 \mathrm{mM} \mathrm{CaCO}_{3}$ and $30 \mathrm{mM}$ GDL) to keep the $\mathrm{pH}$ constant during network formation. The calcium alginate networks formed via internal setting varied in the $R$-value from 0.5 to 2 where the $R$-value is defined according to

$$
R=\frac{2\left[\mathrm{Ca}^{2+}\right]}{\text { guluronate }]}
$$

Externally set alginate gels were prepared by coating the internal wall of a glass beaker $(V=50 \mathrm{ml}$ and $d=40 \mathrm{~mm})$ with alginate by brushing the internal wall with alginate solution, which then was allowed to dry in an oven set at $383 \mathrm{~K}$ for 30 minutes. The proceeding was repeated three times. A volume of $20 \mathrm{ml}$ alginate solution $(1.8 \% \mathrm{w} / \mathrm{w})$ at $293 \mathrm{~K}$ was poured into the glass beaker. The surface of the polymeric solution was sprayed with $0.5 \mathrm{M} \mathrm{CaCl}_{2}$ solution until a gel membrane was formed on top of the alginate solution. The gel membrane was left to set for 30 minutes after which $\mathrm{CaCl}_{2}$ solution at $293 \mathrm{~K}$ was carefully poured on top of the membrane. The $\mathrm{CaCl}_{2}$ solution was left to diffuse through the membrane and into the alginate solution for $48 \mathrm{~h}$ prior to the use of the gel. The $R$-value of the gels varied between 1 and 8 . The $R$-value was varied by the addition of different volumes of $\mathrm{CaCl}_{2}$ solutions at a concentration of $0.5 \mathrm{M}$.

Large deformation rheology. Uniaxial compression tests were performed on all gels using an Instron mechanical test frame (model 5565A). At least three repeats were done for each sample. In the case of the internally set alginate the gel cylinders tested were carefully removed from the mould and aligned in the centre of stainless steel compression plates, which were lubricated with mineral oil to reduce friction. In the case of the externally set gel, the gel was carefully removed from the beaker, after which the membrane was removed using a razor blade and cylinders were stamped out from the top part of the gel (after removal of the membrane). Each gel was carefully examined for cracks or deformation obtained from handling prior to testing. The gels were aligned in the center of stainless steel compression plates as for the internally set alginate gels. A displacement rate of $7 \mathrm{~mm} \mathrm{~min}^{-1}$ was used and the test was performed at room temperature. True stress was calculated using eqn (2)

$$
\sigma=F H / A_{0} H_{0}
$$

and the true strain was calculated from eqn (3)

$$
\gamma=\ln \left(H / H_{0}\right)
$$

with $F, H, A_{0}$ and $H_{0}$ being the force used to compress the sample, the height of the sample, the initial area and initial height of the sample. Creep tests were performed by compressing the samples to 5, 10 and $20 \%$ strain using an initial crosshead speed of $4 \%$ strain per second. The stress response upon relaxation of the gel was studied for up to 300 seconds. The tendency of the gels to return to their initial shape was recorded immediately after the compression force was released as well as upon 2 minutes rest.

CLSM-FRAP Protocol. The confocal laser scanning microscope (CLSM) system used consists of a Leica SP2 AOBS (Heidelberg, Germany) with a $20 \times, 0.5$ NA water objective, with the following settings: $256 \times 256$ pixels, a zoom factor of 4 (with a zoom-in during bleaching), and $800 \mathrm{~Hz}$, yielding a pixel size of $0.73 \mu \mathrm{m}$ and an image acquisition rate of two images per second. The FRAP images were stored as 12 bit tif-images. The $488 \mathrm{~nm}$ line of an argon laser was utilized to excite the fluorescent probes. The beam expander was set to 1 , which lowered the effective NA to approximately 0.35 and yielded a slightly better bleaching and a more cylindrical bleaching profile. The bleached areas are called regions of interest (ROI) in due course of the paper, and were $30 \mu \mathrm{m}$ large discs (nominal radius $r_{\mathrm{n}}=15$ $\mu \mathrm{m})$ at $50 \mu \mathrm{m}$ into the sample. The measurement routine consisted of 20 prebleach images, 1 bleach image - gaining an initial bleaching depth $\approx 35 \%$ of the prebleach intensity in the ROI and 50 postbleach frames, recording the recovery. The FRAP data got normalized by the prebleach fluorescence intensity. It is known that fluorescein does not follow first order bleaching kinetics and would need to be compensated with an actual apparent bleaching intensity distribution, but it was shown that for low enough spatial resolution and large enough ROI $(>5 \mu \mathrm{m}$ with the objective used) those effects are negligible. ${ }^{\mathbf{4 0 4 1}}$

The free diffusion coefficients $D_{0}$ of the probes in the absence of alginate were determined at $298 \mathrm{~K}$. The probes were dissolved in $\mathrm{CaCl}_{2} / \mathrm{CaCO}_{3}+\mathrm{GDL}$ solutions corresponding to the respective $R$-values. $7 \mu$ l of the probe solutions were placed into secure-seal spacer grids between two cover glass slides, and the FRAP measurements were carried out on such locked samples. Hydrodynamic radii $\left(r_{\mathrm{H}}\right)$ - gained using Stokes-Einstein relation - of the used FITC-dextran probes range between $\mathrm{rH} \approx 3.5 \mathrm{~nm}$ for $10 \mathrm{kDa}$, $\mathrm{rH} \approx 8.5 \mathrm{~nm}$ for $70 \mathrm{kDa}$ and $\mathrm{rH} \approx 14 \mathrm{~nm}$ for $500 \mathrm{kDa} \mathrm{MW}$.

To conduct FRAP measurements in the internally set gels corresponding amounts of FITC-dextran were introduced in the alginate solution before the addition of calcium to yield a probe 
concentration of $100 \mathrm{ppm}$ in the gelled system. For the externally set gels the FITC-dextrans were introduced in two ways: (a) as described above by mixing it into the polymer solution; (b) FITC-dextrans were dispersed into the $\mathrm{CaCl}_{2}$ solution above the membrane and diffused into the alginate sol together with the cross-linking ions. The probe concentration was chosen to be in the linear regime of the fluorescence dependence on the concentration. ${ }^{\mathbf{4 1}}$

As described above, the capillary gels were set during 2 days. The membrane above the formed capillary gel got removed, and a ca. $2 \mathrm{~cm} \times 2 \mathrm{~cm} \times 2 \mathrm{~cm}$ sized sample got cut out - centrally and directly under the membrane - to perform the FRAP measurement. The surface of the cube, cut perpendicular to the direction of capillary growth, got absorbed on a cover glass slide; then loaded on the microscope stage and FRAP measurements were carried out in the upright mode of the microscope at constant $298 \mathrm{~K}$. At least 6 FRAP measurements have been performed on different spatial coordinates per sample. The ROIs' spatial locations have been chosen, such that in the whole field of view $(\approx 200 \mu \mathrm{m} \times 200 \mu \mathrm{m})$ no capillary was present. This means that the diffusion in the bulk phase between the capillaries got tested. To test the reproducibility every sample has been remade at least once. All of the recorded recoveries were quick enough to yield Gaussian intensity distributions in the initial recovery images within the bleached area/ROI. Therefore the FRAP model called "most likelihood estimation for FRAP data with a Gaussian starting profile"41 is valid for evaluation of the data. A script provided by Jonasson et al. ${ }^{41}$ was utilized to analyze the data within this framework in Matlab, Mathworks, U.S.A.

Embedding and transmission electron microscopy. Small gel cubes, $1 \mathrm{~mm} \times 1 \mathrm{~mm} \times 1 \mathrm{~mm}$, were cut out of the gel and fixed in $2 \%$ glutaraldehyde containing $0.1 \%$ Ruthenium $\operatorname{Red~} \mathrm{CaCl}_{2}$. The $\mathrm{CaCl}_{2}$ concentration corresponded to the concentration in the gel. Dehydration was performed in a graded ethanol series ending with propylene oxide prior to the TLV resin infiltration. The exchange of water during the dehydration step is performed gradually in order to minimize shrinkage; any occurring shrinkage is assumed to be isotropic and not to change the characteristics of the microstructure. The samples were embedded in epoxy resin TLV and polymerized for 20 hours at $333 \mathrm{~K}$. Ultrathin sections around $60 \mathrm{~nm}$ were cut with a diamond knife using an ultramicrotome (Powertome XL, RMC products, Boeckeler Instruments Inc, Tucson, Arizona). The ultrathin sections were placed on 400 mesh gold grids and stained with periodic acid, thiosemicarbazide and silver proteinate. Images of the alginate gel were recorded with a transmission electron microscope (TEM) LEO 706E (LEO Electron Microscopy Ltd., Oberkochen, Germany) at a $80 \mathrm{kV}$ accelerating voltage.

Image analysis of gel strands and voids. Gel strands and voids must be segmented to measure different relevant features of the images. Here, voids are defined as the spaces in-between the gel strands in the gel network that are available for the dextran diffusion. To process the TEM images and yield a binary representation of the network strands, the first steps were to remove uneven illumination using low-pass filtering and image multiplication, and to perform Wiener filtering to reduce the amount of noise and to enhance the gel strands. Subsequently, morphological Top-Hat transformation was used to increase contrast and manual thresholding was performed to segment gel strands and voids. Finally, small objects were removed and a morphological close operation was applied to connect adjacent objects. The image analysis was performed in Matlab, Mathworks, USA. We analyzed TEM images of the externally and internally set gels; for the externally set gels only the structures characterizing the bulk network were recorded in between the capillary zones. As both gels have the same effective polymer concentration the manual thresholding was applied in such a manner, that the area fraction of the gel strands was constant for all analysed images. The resulting binarized images were then used for image analysis. We calculated the volume, weight, and mean volume for pores and interfacial area applying the algorithm by Gundersen and co-workers ${ }^{\mathbf{4 2}}$ on the binary images. For each gel type six images acquired randomly at different locations in the gel were analyzed. Micrographs got analysed at one constant magnification of $10000 \times$.

Image analysis of the pore diameter. The gels were died by soaking them a few minutes in a solution containing $0.75 \mathrm{mg}$ ruthenium $\mathrm{red} / \mathrm{ml}$ water (milliQ). The capillaries were visualized using a light microscope, Aigo Digital Viewer GE-5, and pore diameter and number were analysed using the program ImageJ.

\section{Results and discussion}

\section{Macrostructure of calcium induced capillary gels}

Several studies have been performed on the effect of the type of (heavy divalent) ions, ion concentration and $\mathrm{M} / \mathrm{G}$ ratio of the alginate used on the diameter size of the capillary as well as on the capillary density. ${ }^{23,43}$ In general, an alginate with high G content gives rise to smaller capillaries and a higher capillary density compared to an alginate high in $\mathbf{M}$ units. ${ }^{7}$ Furthermore, while the capillary diameter and density will vary with the type of (heavy metal) ion added, increasing the concentration of ions leads to reduced capillary diameter and a higher capillary density. ${ }^{23}$ Fewer studies have been performed on capillary formation in alginate gels using calcium. However, the results obtained in this study show relatively well defined capillaries which are homogeneously distributed over the crosssection of the gel surface, as displayed in Fig. 1(a). Image analysis of such gels results in an initially reducing capillary diameter with increasing total amount of $\mathrm{Ca}^{2+}$, and thus increasing $R$-value, after which the capillary diameter appears to be less dependent on $R$. However, the capillary density increases consistently with the $R$ value, at least for the values studied here (Fig. 1(b)). The $R$-value of the final gel is varied by adding different volumes of a fixed electrolyte solution $\left(0.5 \mathrm{M} \mathrm{CaCl}_{2}\right)$. The capillary diameter obtained varies between 60 and $110 \mu \mathrm{m}$ depending on the final $R$-value. The capillary diameter of 60 to $70 \mu \mathrm{m}$ that are obtained for the higher $R$-values of $4-8$ is in relative agreement with the findings of Despang and co-workers of a capillary diameter of $43 \mu \mathrm{m}$ using a high $\mathrm{G}$ alginate and $1 \mathrm{M} \mathrm{CaCl}_{2}$ as an electrolyte. ${ }^{7}$ It is worthwhile to note that no capillaries were formed below a $R$-value of 2 , thus indicating that the capillary formation takes 


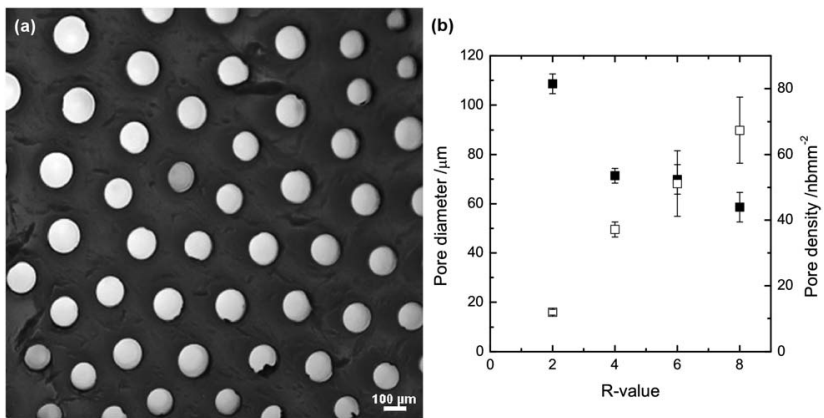

Fig. 1 (a) Cross-section of a capillary gel dyed with ruthenium red at $R=2$. (b) Pore diameter (solid symbols) and density (open symbols) as a function of the $R$-value; formed at $293 \mathrm{~K}$.

place only once excess $\mathrm{Ca}^{2+}$ is available - above crosslinking saturation of the alginate $(R=1)$.

\section{Rheological differences between isotropic and capillary gels}

Stress relaxation tests have been performed on capillary and internally set calcium alginate gels of $R=2$ and at room temperature. A fixed strain of 5,10 and $20 \%$ was applied and the stress required to maintain the strain at the specific value was recorded over time, as depicted in Fig. 2. Both types of the gel show viscoelastic behaviour as the stress required to maintain a strain of 5,10 and $20 \%$ respectively varies initially, while levelling out to a close to constant nonzero value ${ }^{14}$ at times $>200$ seconds. It is observed that less pressure is required in order to maintain the capillary gel at a given strain compared to the internally set gels. The modulus $G$ of the material $(G(t)=$ stress $(t) /$ strain) indicates that the linear region for the modulus as a function of strain ends somewhere between 10 and $20 \%$ strain. This is in agreement with literature data reporting linearity up to at least $17 \%$ strain for internally set calcium alginate gels. ${ }^{14}$ Comparing the moduli, depicted in Fig. 2(c), of $38 \pm 4$ $\mathrm{kPa}$ obtained at 5 and $10 \%$ strain for the internally set gel of $R=$ 2 shows reasonable agreement with previously reported values for high guluronate alginate in excess of calcium. ${ }^{14}$ The results show further that the moduli for the capillary gels of $6.2 \pm 0.7$ $\mathrm{kPa}$ at 5 and $10 \%$ strain are considerably lower compared to the internally set gels despite similar composition (calcium and alginate concentration) of the two types of gels. It should further be stated that additional stress relaxation tests at different strains should be done in order to specify the linear region for the capillary gels. Observing the tendency for the gels to recover their initial height, after the external load has been withdrawn, is displayed in Table 1. These data indicate a higher degree of plasticity for the capillary gels.

Rupture strength is additionally used to characterise the mechanical properties of the two differently set gels at varying $R$-values. Fig. 3(a) depicts the results obtained for the internally set gels. The isotropic calcium alginate gels show an increase in rupture strength as the $R$-value is increased to $R=1$, above which the rupture strength levels out as $R$ is increased to $R=2$. It has been suggested that increasing calcium concentration for a fixed alginate concentration initially increases the length of the junction zones (up to $R=1$ ), leading to an increase in rupture strength. ${ }^{14,15}$ Above the stoichiometric value of $R=1$, no further junctions are formed, which could explain the apparent levelling out of rupture strength as $R$ goes from 1 to 2 for the internally set gels. A similar explanation could be given for the capillary gels where no increase in rupture strength is observed as $R$ is increased from 2 to 8 .

The results show that the rupture strength of the internally set gel of $R \geq 2$ is considerably higher compared to the rupture strength of the capillary gels (see Fig. 3(b)) at equal $R$ and alginate concentration. It is further noticeable that accounting for only the actual gel area (thus subtracting the area of the capillary pores from the total area of the crosssection) only increases the rupture strength to a small extent. The capillary gel still has a low rupture strength compared to the internally set alginate gel. The rupture strength data follow the results from the stress relaxation test that the mechanical properties of the differently set gels vary considerably despite same composition. The large difference observed between the internally set gel and the capillary gel suggests that the gelation process as such introduces differences also within the microstructure of the gel, i.e. it is not only the macroscopic difference (presence of capillaries) that influences the mechanical properties of the two differently set gels. However, this needs to be confirmed further.
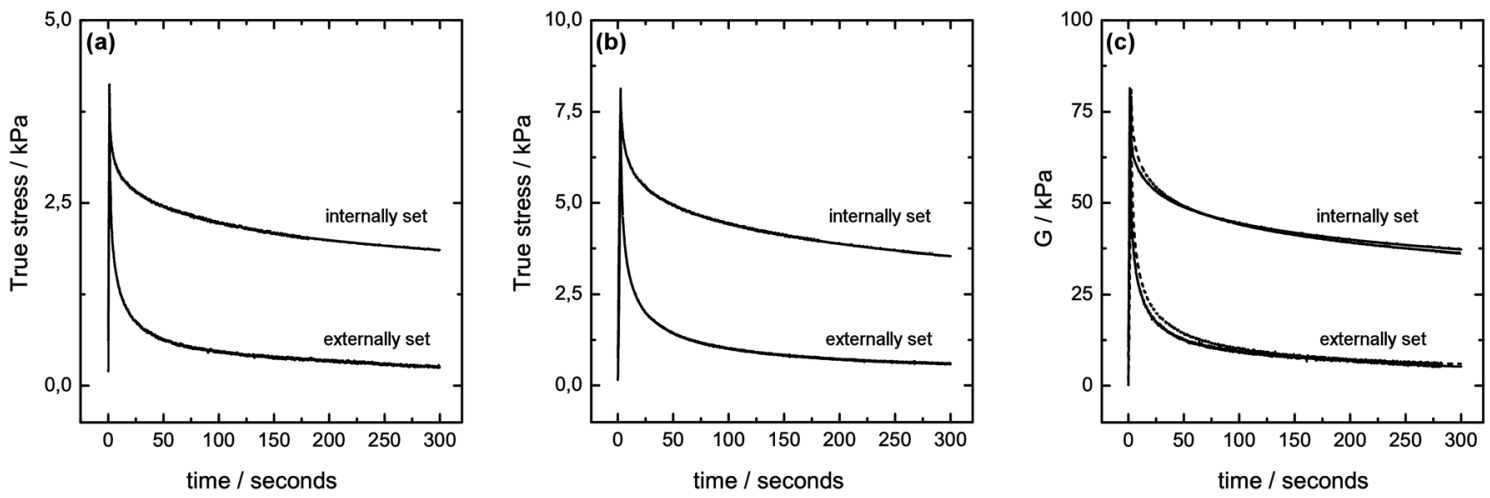

Fig. 2 Stress relaxation of calcium alginate gels with a $R$-value of 2 prepared via internal gelation and external gelation when compressed (a) to $5 \%$ strain and (b) to $10 \%$ with an initial crosshead speed of $4 \% /$ second, (c) stress relaxation for the modulus G: $5 \%$ strain (solid line) $\& 10 \%$ strain (dashed line) for the internal external gelation method. 
Table 1 Recovery in percentage of initial height of random and capillary gels at different degrees of compression and times. The gels had an initial height of $10 \mathrm{~mm}$, a diameter $10 \mathrm{~mm}$ and a $R$-value of 2

Compression $/ \%$

5

\begin{tabular}{|c|c|c|c|c|c|c|}
\hline \multirow[b]{2}{*}{ Time } & \multicolumn{2}{|l|}{5} & \multicolumn{2}{|l|}{10} & \multicolumn{2}{|l|}{20} \\
\hline & Random & Capillary & Random & Capillary & Random & Capillary \\
\hline Immediately after removal of force & 100 & 91 & 96 & 92 & 91 & 84 \\
\hline
\end{tabular}
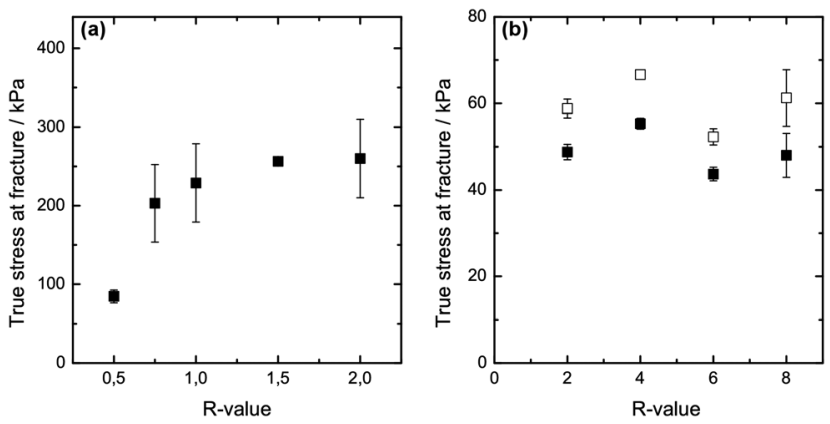

Fig. 3 True stress at rupture for uniaxial compression (solid symbols) as a function of the $R$-value for (a) internally set and (b) externally set alginate gels at a polymer concentration of $1.8 \%$ and $293 \mathrm{~K}$; open symbols correspond to the cylinder area corrected for pore capillary surface of the cross-section.

\section{Probe-diffusion in isotropic and capillary gels}

To study the diffusivity of different molecular weight FITCdextrans in the bulk phase of internally and externally set alginate gels we performed a series of FRAP measurements. All studied networks have, as described above, the same effective polymer concentration (yielded by the subtraction of the pore areas) of $2 \% \mathrm{w} / \mathrm{w}$. Fig. 4 displays a typical FRAP recovery, showing the time evolution of the fluorescent intensity. A microscopy image during a typical FRAP measurement on an externally set gel is shown in Fig. 4(b), and visualizes a homogeneous fluorescent distribution over the whole field of view of around $200 \times 200 \mu \mathrm{m}$; that means that the capillaries are well separated from the spatial region of the measurement, and with probes sized $>5 \mathrm{~nm}$ (and a self diffusion coefficient $<70 \mu \mathrm{m}^{2}$ $\mathrm{s}^{-1}$ ), will not influence the recovery curves recorded during the experiments in the time period of $25 \mathrm{~s}$. In comparison, Fig. 4(c) illustrates how a typical capillary would be visualized during a FRAP measurement.

\section{Effect of available calcium}

As seen above, varying the $R$-value results in gels of different strength and elasticity at values up to $R=1$, and constant mechanical properties at $R$-values above 1 . However, it appears that not only the $R$-value itself determines the rheology, but also the method of gelation - internal or external ion addition influences the functionality strongly. Here, we investigate the influence of the $R$-value on the probe diffusion of 70 kDa FITCdextran, a probe sized $\approx 17 \mathrm{~nm}$ (recalculated diameter from the hydrodynamic radius). Fig. 5 displays $R$-value sweeps and the corresponding normalized diffusion coefficients, for the calcium ranges in which gelation was possible. In internally set gels the diffusivity of the probes is reduced by $80 \%$ compared to the free diffusion coefficients $D_{0}$ of the probes in the absence of alginate. Variations in the $R$-value between 0.4 and 2 did not cause significant changes in the probe diffusion. The bundle size of the network strands is known to be increasing for increasing $R$-values up to $R=1$, when enough junction zone forming $\mathrm{Ca}^{2+}$ is available to incorporate all $\mathrm{G}$ units into aggregates via the egg-box mechanism. ${ }^{13}$ Our results indicate that although the network bundle size is increasing, the pore sizes being tested by FITC-dextran diffusion are not influenced by the strand thickness. However, the effect of polymer concentration seems to be the dominating factor for the diffusion. Similarly are the findings depicted in Fig. 5(b) for the externally set gels. No significant differences regarding the diffusivity of $70 \mathrm{kDa}$
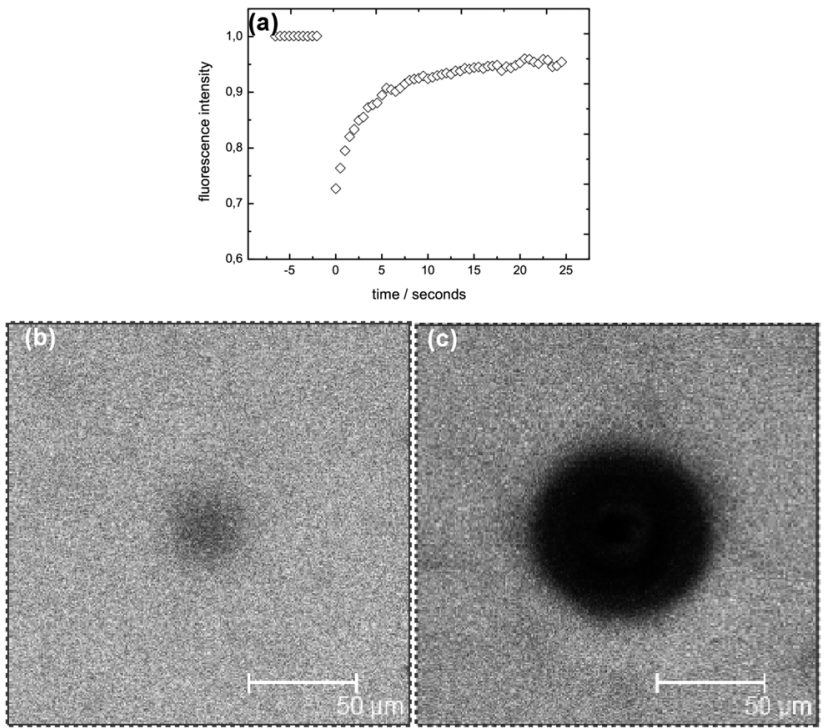

Fig. 4 (a) Example of the time evolution of a FRAP recovery for $10 \mathrm{kDa}$ FITC-dextran in an externally set gel. CLSM images: (b) image of a typical postbleach during a FRAP recovery measurement. Before recording the FRAP recovery, in the middle of the image in a region of 30 micron diameter ca. 30\% of the probes got photobleached; resulting in a drop of the recorded intensity. (c) Image of a capillary zone, recorded with the same imaging settings as utilized for a FRAP measurement, to visualize that capillaries could be identified under the CLSM. FRAP measurements were NOT carried out at such spatial locations. 

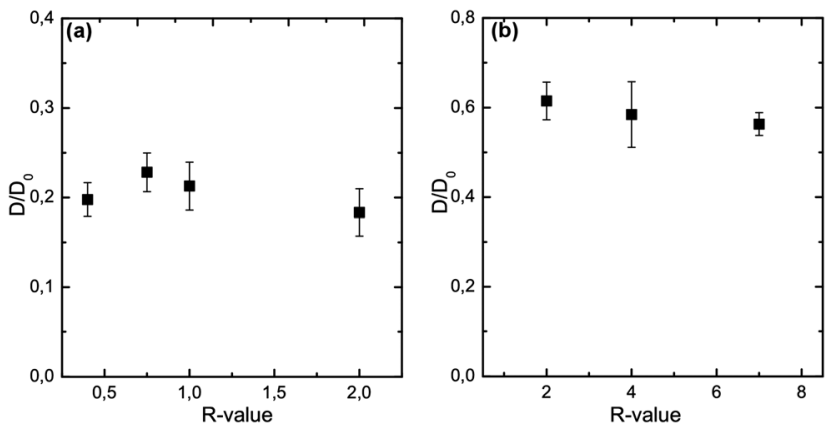

Fig. 5 Normalized diffusion coefficient depending on the $R$-value for $70 \mathrm{kDa}$ FITC-dextran (a) in $2 \% \mathrm{w} / \mathrm{w}$ internally set gels and (b) in $2 \% \mathrm{w} / \mathrm{w}$ externally set gels. The probes were added via the external-diffusive mechanism, by mixing the probes in the $\mathrm{CaCl}_{2}$ solution above the gelmembrane.

FITC-dextran could be found over the whole $R$-value range. We find a reduction of the diffusivity by $40 \%$ for $70 \mathrm{kDa}$ FITCdextran in externally set gels. Interestingly, testing the microstructure of alginate gels of the same effective polymer concentration with $\approx 17 \mathrm{~nm}$ sized probes a highly significant difference between the two gel setting methods is found. The externally set gels exhibit a diffusivity more than twice as big as for the internal set gels. These results suggest a different network microstructure and/or connectivity for the two respective gel setting methods.

\section{Effect of probe size}

To elucidate the difference in diffusivity for the two types of gelation in more detail, we studied the diffusion of FITC-dextran of increasing molecular weight in externally and internally set gels at $R=2$, a $R$-value at which both gelation methods coexist. This approach allows testing the obstruction properties and connectivity of the alginate networks, when penetrated by slightly smaller and larger probes than 70 kDa FITC-dextran. Fig. 6 shows the results of FRAP measurements on a molecular weight series in both types of alginate gels. For both gels same general trends are observed: increasing the molecular weight decreases the diffusivity, as probes of higher hydrodynamic radius do feel a stronger hindrance of their molecular motion. We find for $10 \mathrm{kDa}$ FITC-dextran a reduction of the diffusivity by $25 \%$ and $35 \%$ for the external and internal methods, respectively. This means that $7 \mathrm{~nm}$ sized probes are not freely diffusing any more and are partly hindered by the network strands. This statement is true for both gelation methods, yet the internally set gel has a slightly higher sieving effect. For the largest probe, 500 kDa FITC-dextran, a strong retardation of the diffusivity of $>80 \%$ is observed for both gel types. Also, the FRAP recovery was found not to reach the initial fluorescent intensity; and a corresponding immobile fraction of $50 \%$ is observed. This means that only $50 \%$ of the probes are mobile and contribute to the recovery, whereas the other probes are physically locked in and do not move in the time scale of the measurement (for 10 and $70 \mathrm{kDa}$ FITC-dextran no immobile fraction is observed). For $500 \mathrm{kDa}$ dextrans we do observe the same retardation and immobile fraction irrespective of the gelation method. This indicates that the probes are

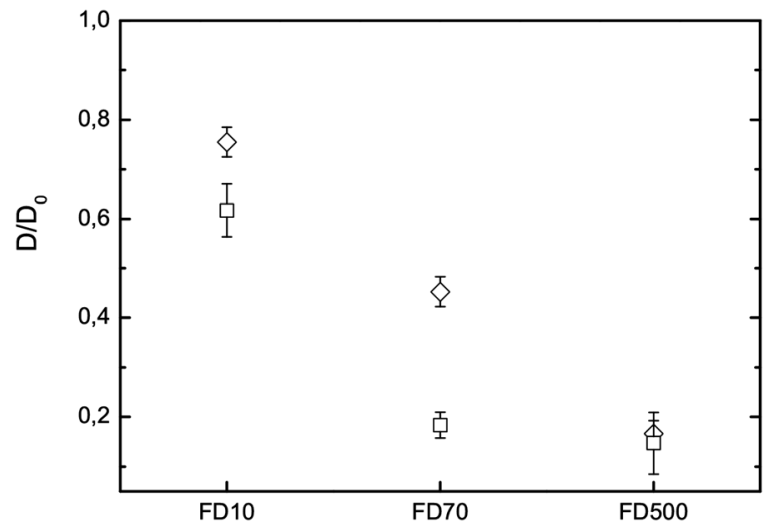

Fig. 6 Normalized diffusion coefficient for $10 \mathrm{kDa}$ (FD10), $70 \mathrm{kDa}$ (FD70) and $500 \mathrm{kDa}$ (FD500) FITC-dextrans at $R=2$. (Diamond) externally set gel and (square) internally set gel. Probes were mixed in the alginate solution before gelation.

clogging up many pores of the network and the probe size of $\approx 28$ $\mathrm{nm}$ is the dominating factor in $2 \% \mathrm{w} / \mathrm{w}$ alginate gels, irrespective of the gel setting method. The diffusivity for $70 \mathrm{kDa}$ FITC-dextran yields the biggest difference for the two gelation methods. Utilizing this probe reduces the diffusivity by $80 \%$ for the internal gelation method, compared to reduction of only $55 \%$ via the external method. Thus, the differences in porosity and connectivity of the network structures are the strongest as examined by tracers sized $\approx 17 \mathrm{~nm}$.

\section{Effect of probe introduction}

Also, comparing the results for $70 \mathrm{kDa}$ FITC-dextran in the externally set gel, depicted in Fig. 5 and 6 , a deviation is found. This arises from the fact that for the set of experiments in Fig. 5 the fluorescent probes were added via the external method, together with the $\mathrm{Ca}^{2+}$-ions. In contrast, the measurements in Fig. 6 show the case in which probes were mixed in the alginate solution. Comparing the diffusivity of $70 \mathrm{kDa}$ FITC-dextran in externally set gels at $R=2$ a $15 \%$ stronger reduction is found compared to the case when the probes are added in the alginate solution. Dextrans mixed into the polymeric solution are homogeneously distributed throughout the sol, thus suggesting, as the gelation sets in and forms heterogeneous network structures, that probes will be equally likely located in tighter network structures as well as in more porous regions. Adding the probes via a diffusive mechanism during the gelation yields an overall faster diffusion in the set gels and indicates that the probes were not able to penetrate the whole network structure equally, and thereby weighted the diffusion in the open network structures excessively. However, both methods of probe introduction resulted in a significant difference in the diffusivity between the externally and internally set gels. The addition of the probes in the alginate solution should be preferred in future studies to gain well distributed tracers throughout all hierarchies of network structures.

\section{Microstructures of the bulk vs. capillary wall}

Interesting information about differences and similarities between polysaccharide gels can be obtained via transmission 
electron microscopy (TEM). ${ }^{19,44}$ TEM micrographs of the bulk network structures of alginate gels at $R=2$ are shown in Fig. 7 . Similarly to the FRAP section, care is taken to image the gel phase in between the capillaries and not in their vicinity. For both gelation methodologies, we observe gel networks consisting of polymer strands (dark threads) and voids (light grey space), the latter being in the range of $50 \mathrm{~nm}$ and above. These structures are similar in character to other physical gels such as pectins and carrageenans. ${ }^{18,19}$ The micrographs for the externally and internally set gels have a heterogeneous network with a wide range of pore sizes, with largest pores in the range of 200 $\mathrm{nm}$ as well as dense network clusters. However, the gel network in the vicinity to the capillary walls is strongly affected by capillary formation and it could be hypothesised that such structures are formed due to a hydrodynamic flow field as suggested in the model by Treml and Kohler. ${ }^{45}$

Fig. 9 displays the microstructure in proximity to the capillary zones of an externally set gel at different magnifications. Here, some interesting features can be observed. The boundary area between the capillaries and the network is not sharp, thus there is no distinct interfacial layer. The interface between the network and the capillary consists of porous structures, and micron sized aggregates of dangling network chains reach into the capillary region. Further, the network in proximity of the capillary exhibits an anisotropic structure and chains seem to be elongated in the tangential direction of the macro-pore. This structure is distorted with a lower connectivity than the interior

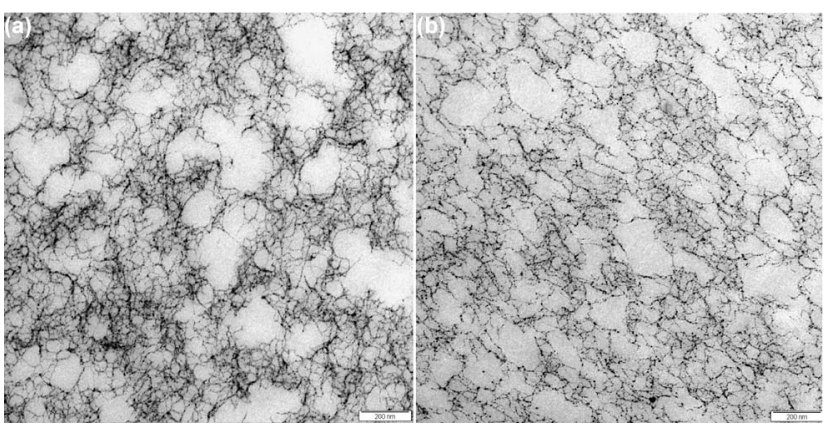

Fig. 7 TEM micrographs of alginate gels at $R=2$; (a) micrograph of the bulk network in an externally set gel and (b) internally set gel.

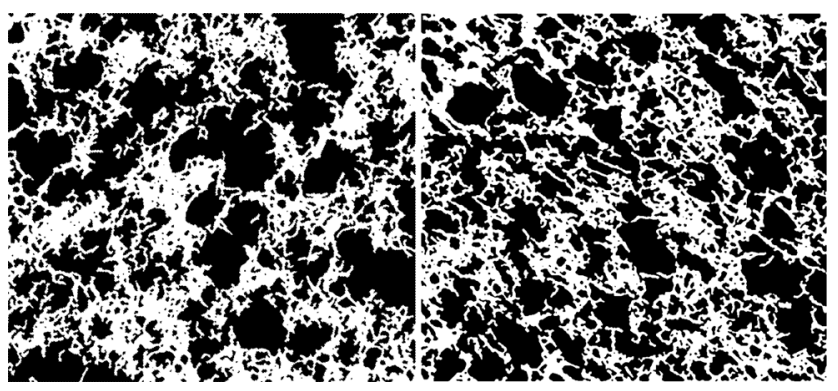

Fig. 8 Binarized images of the TEM micrographs displayed in Fig. 7 (Left) externally set and (right) internally set. The images were cropped to not contain the scale bars during the image analysis.

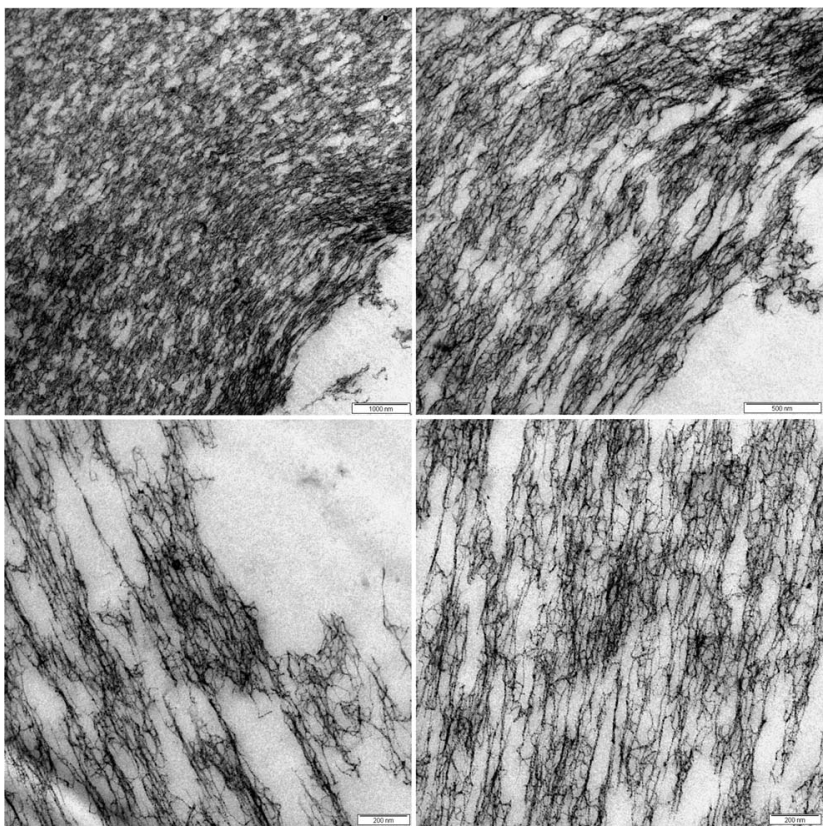

Fig. 9 TEM micrograph of an externally set alginate gel at $R=2$ in proximity to the capillary zones at different magnifications.

of the network as observed in Fig. 7. It reaches 5-10 micrometers into the structure and takes up considerable volume. This part of the structure will most likely contribute to the rheological properties of the gels and could be one of the reasons for the higher plasticity of the capillary gels.

Image analysis was performed to determine the pore sizes of the alginate gels on binarized TEM micrographs. Following the routine described in the materials and methods section, examples of such gained binary images are illustrated in Fig. 8 . Analysing the volume weight mean volume of the pores in the bulk network structures of externally and internally set gels, we find pore volume values corresponding to spheres of diameter $d$ for the externally set gel: $d_{\text {external }}=59.5 \pm 8.5 \mathrm{~nm}$, and respectively $d_{\text {internal }}=56.8 \pm 3.9 \mathrm{~nm}$ for internally set gel. Thus, the pore volumes gained via the image analysis of roughly $60 \mathrm{~nm}$ thin sections do not significantly differ for the two gelation methods. However, as pointed out the overall network morphology of many physical gels are similar even if the gelation mechanism and properties may differ. ${ }^{18,19}$ Here other features contribute to the properties such as the order and strength of the gel strands, interactions between strands as well as the connectivity and heterogeneity of the network.

The results presented show that the way alginate gels are prepared (via internal or external addition of calcium) do not only give rise to the intriguing differences in macroscopic appearance of the gels, by introducing open capillaries, but also changes the gel network structure between the capillaries. These changes give rise to a network structure that oppose less hindrance to FITC - dextrans of sizes 10 and $70 \mathrm{kDa}$ compared to internally set gel. It is worthwhile to note that the differences introduced via the route of calcium addition is larger than what could be obtained by variation of the available amount of 
calcium below and above the stoichiometric amount, thus varying the number and length of junction zones as well as their degree of aggregation. The plasticity and lower moduli of the externally set alginate gels further suggest the presence of a network where the external force is suboptimally distributed and breakage or irreversible aggregation of the network occurs at lower strain. The distorted and anisotropic zone in the vicinity of the capillaries will certainly contribute to the rheological behaviour of the capillary gels but further studies will focus on other differences in the fine structures of the gel networks.

\section{Conclusions}

Starting with identical compounds - quantity of alginate and calcium - we are able to tune the elasticity/plasticity and the rupture strength of formed gels in one order of magnitude between 10s and 100s of Pa. Additionally we can tailor the mass transport of nanometre sized diffusants by the choice of the gelation methodology. The diffusivity for dextrans sized smaller than $20 \mathrm{~nm}$, determined in the gel phase where the influence of capillaries is negligible, is higher in alginate gels formed via the external gel method compared to the internal methodology. Both gel types do hinder larger diffusants. The results indicate that the gelation methodology will not only influence the macroscopic structure, via the formation of parallel aligned capillaries as is the case of externally set alginate gels, but also the microstructure. The TEM images showed a 5-10 micrometer wide zone close to the capillary wall that had an anisotropic and distorted structure with a lower connectivity. This weaker structure can contribute to the higher plasticity and lower moduli of the capillary gels. The mean pore sizes found on the inner parts of the gels did not depend on external or internal addition of crosslinking ions. To explain the difference of diffusivity further studies of the fine structure and the connectivity of the gels in three dimensions will be needed.

\section{Acknowledgements}

The authors thank Krassimir Velikov for encouragement and helpful discussions. We thank Linda Persson and Jonathan Jonson for supply with capillary gels and help with the rheological measurements. We are grateful for the financial support from SuMo Biomaterials and Vinnmer grant to A.S.

\section{References}

1 K. Draget, in Handbook of hydrocolloids, ed. G. O. Phillips and P. A. Williams, Woodhead Publishing Ltd, 2nd edn, 2009, pp. 807-828.

2 M. Rinaudo, Polym. Int., 2008, 57, 397-430.

3 S. Thomas, J. Wound Care, 2000, 9, 56-60.

4 H. L. Lai, A. Abu'Khalil and D. Q. Craig, Int. J. Pharm., 2003, 251, 175-181.

5 M. Figueira, B. Volesky, V. Ciminelli and F. A. Roddick, Water Res., 2000, 34, 196-204.

6 F. Lim and A. M. Sun, Science, 1980, 210, 908-910.
7 F. Despang, A. Borner, R. Dittrich, G. Tomandl, W. Pompe and M. Gelinsky, Materialwiss. Werkstofftech., 2005, 36, 761-767.

8 G. Miralles, R. Baudoin, D. Dumas, D. Baptiste, P. Hubert, J. Stoltz, E. Dellacherie, D. Mainard, P. Netter and E. Payan, J. Biomed. Mater. Res., 2001, 57, 268-278.

9 K. Y. Lee and D. J. Mooney, Prog. Polym. Sci., 2012, 37, 106-126. 10 T. Andersen, J. E. Melvik, O. Gåserød, E. Alsberg and B. E. Christensen, Biomacromolecules, 2012, 13, 3703-3710.

11 Y. Cheng, X. Luo, G. F. Payne and G. W. Rubloff, J. Mater. Chem., 2012, 22, 7659-7666.

12 E. R. Morris, D. A. Rees, D. Thom and J. Boyd, Carbohydr. Res., 1978, 66, 145-154.

13 B. T. Stokke, K. I. Draget, O. Smidsrød, Y. Yuguchi, H. Urakawa and K. Kajiwara, Macromolecules, 2000, 33, 1853-1863.

14 J. Mitchell and J. Blanshard, Journal of Texture Study, 1976, 7, 219-234.

15 J. Zhang, C. R. Daubert and E. A. Foegeding, J. Food Sci., 2005, 70, e425-e431.

16 J. Zhang, C. R. Dauber and E. A. Foegeding, J. Food Eng., 2007, 80, 157-165.

17 D. Bernin, G. J. Goudappel, M. van Ruijven, A. Altskar, A. Strom, M. Rudemo, A. M. Hermansson and M. Nyden, Soft Matter, 2011, 7, 5711-5716.

18 C. Löfgren, S. Guillotin and A.-M. Hermansson, Biomacromolecules, 2006, 7, 114-121.

19 A. M. Hermansson, in Food materials science: principles and practice, ed. J. Aguilera and P. Lillford, Springer Verlag, 2008, Structuring water by gelation, ch. 13, pp. 255-280.

20 A. Strom and M. A. K. Williams, J. Phys. Chem. B, 2003, 107, 10995-10999.

21 K. I. Draget, G. Skjåk-Bræk and O. Smidsrød, Int. J. Biol. Macromol., 1997, 21, 47-55.

22 A. Mikkelsen and A. Elgsaeter, Biopolymers, 1995, 36, 17-41. 23 H. Thiele and K. Hallich, Colloid Polym. Sci., 1957, 151, 1-12.

24 R. Dittrich, G. Tomandl, F. Despang, A. Bernhardt, T. Hanke, W. Pompe and M. Gelinsky, J. Am. Ceram. Soc., 2007, 90, 1703-1708.

25 F. Despang, R. Dittrich and M. Gelinsky, in Advances in Biomimetics, ed. A. George, InTech, Rijeka, 2011, Novel biomaterials with parallel aligned pore channels by directed ionotropic gelation of alginate: mimicking the anisotropic structure of bone tissue, ch. 17, pp. 349-72.

26 J. Thumbs and H.-H. Kohler, Chem. Phys., 1996, 208, 9-24.

27 H. Treml, S. Woelki and H.-H. Kohler, Chem. Phys., 2003, 293, 341-353.

28 J. E. Maneval, D. Bernin, H. T. Fabich, J. D. Seymour and S. L. Codd, Magn. Reson. Chem., 2011, 49, 627-640.

29 H. Frisch, T. Wang and T. Kwei, J. Polym. Sci., Part A-2, 1969, 7, 879-887.

30 I. Prigogine, Science, 1978, 201, 777-785.

31 A. Onuki and S. Puri, Phys. Rev. E: Stat. Phys., Plasmas, Fluids, Relat. Interdiscip. Top., 1999, 59, R1331-R1334.

32 K. Furusawa, S. Sato, J.-i. Masumoto, Y. Hanazaki, Y. Maki, T. Dobashi, T. Yamamoto, A. Fukui and N. Sasaki, Biomacromolecules, 2011, 13, 29-39. 
33 B. Amsden and N. Turner, Biotechnol. Bioeng., 1999, 65, 605610.

34 M. Golmohamadi and K. J. Wilkinson, Carbohydr. Polym., 2013, 94, 82-87.

35 Y. Cheng, R. K. Prud'homme and J. L. Thomas, Macromolecules, 2002, 35, 8111-8121.

36 S. Seiffert and W. Oppermann, Polymer, 2008, 49, 4115-4126.

37 J. Hagman, N. Loren and A.-M. Hermansson, Biomacromolecules, 2010, 11, 3359-3366.

38 J. Hagman, Structure Dynamics and Heterogeneity in Soft Materials Determined by FRAP, Ph.D. thesis, Chalmers University of Technology, 2012.

39 A. Strom, Characterisation of pectin fine-structure and its effect on supramolecular properties. Ph.D. thesis, Cork University, 2006.
40 K. Braeckmans, L. Peeters, N. Sanders, S. De Smedt and J. Demeester, Biophys. J., 2003, 85, 2240-2252.

41 J. K. Jonasson, N. Loren, P. Olofsson, M. Nyden and M. Rudemo, J. Microsc., 2008, 232, 260-269.

42 H. Gundersen, T. Bendtsen, L. Korbo, N. Marcussen, A. Møller, K. Nielsen, J. Nyengaard, B. Pakkenberg, F. Sørensen, A. Vesterby and M. West, APMIS, 1988, 96, 379-394.

43 K. S. Khairou, W. M. Al-Ghetami and R. Hassan, J. Membr. Sci., 2002, 209, 445-456.

44 A. M. Hermansson and M. Langton, in Physical Techniques for the study of food biopolymers, ed. S. Ross-Murphy, Chapman and Hall, 1994, Electron Microscopy, ch. 6, pp. 277-341.

45 H. Treml and H.-H. Kohler, Chem. Phys., 2000, 252, 199-208. 